\title{
Simulação e análise de defeitos em nanoaneis de ferro
}

\author{
Sarah de Carvalho Caminha ${ }^{[1]}$, Idalmir de Souza Queiroz Júnior ${ }^{[2]}$ \\ ${ }^{[1]}$ Universidade Federal Rural do Semi-árido; sarahcaminha@outlook.com \\ ${ }^{[2]}$ Universidade Federal Rural do Semi-árido; idalmir@ufersa.edu.br \\ Recebido: 15/04/2019; \\ Aceito: $21 / 08 / 2019$; \\ Publicado: 07/10/2019.
}

Resumo: Nos últimos anos, houve grandes avanços no estudo de propriedades magnéticas de nanoestruturas magnéticas e este tópico ainda continua sendo alvo de pesquisa no campo da física nos dias de hoje. Do ponto de vista teórico, há interesse na análise das propriedades magnéticas em nanoelementos magnéticos por causa da grande possibilidade de aplicações tecnológicas, como sensores e memórias magnéticas. Dentre esses nanoelementos magnéticos, nanoaneis têm sido investigados devido à atratividade de suas propriedades físicas e à potencialidade de sua aplicação em dispositivos funcionais. Nanoaneis magnéticos podem constituir células de memória de alta performance, que podem eficientemente armazenar, gravar e ler informações. No entanto, nanoaneis defeituosos são inevitáveis no caso de produção em massa. Os defeitos presentes nessas nanoestruturas podem afetar as propriedades magnéticas de todo o sistema, além disso, a inserção de defeitos propositais pode resultar em maior controle das fases que surgirão ou de outros parâmetros de interesse. Diante dessas possibilidades, estuda-se nanoaneis isolados de ferro com a presença de defeitos na forma de assimetria. Os nanoaneis são estudados em função da variação dos parâmetros geométricos da espessura do anel e do tamanho da assimetria. O objetivo é investigar as propriedades magnéticas, focando no comportamento do campo coercivo e da magnetização remanescente, e o processo de reversão de magnetização através da histerese magnética e pela configuração dos momentos magnéticos em remanência. Utilizou-se simulação micromagnética, que se baseia na minimização do torque realizado pelo campo magnético efetivo local sobre o dipolo magnético de cada célula de simulação. Os resultados obtidos mostram aumento do campo coercivo com aumento da assimetria; comportamento variado da magnetização remanescente, conforme a configuração, porém com queda abrupta para anéis simétricos; e aumento do campo necessário para saturar, conforme diminui-se o defeito.

Palavras-chave: nanoaneis; propriedades magnéticas; histerese

\section{INTRODUÇÃO}

A crescente necessidade do ser humano de encontrar novas tecnologias cada vez mais eficientes e que sejam passíveis de aplicação torna o estudo do magnetismo relevante, pois através deste campo de estudo, pode-se produzir resultados em diversos campos, como por exemplo nas áreas de tecnologia da informação quântica e de biotecnologia. Uma das aplicações mais simples e antigas, que prova a importância do estudo do magnetismo é a bússola, que auxiliou na descoberta de grandes continentes, mesmo antes de cristo. $\mathrm{O}$ magnetismo é a base para o desenvolvimento tecnológico que pode trazer grandes benefícios para a humanidade, por isso estudar e controlar propriedades magnéticas de sistemas tem sido de grande interesse para pesquisadores, instituições acadêmicas e empresas [1].

Com o avanço científico nas últimas décadas, tornou-se possível produzir e caracterizar amostras em escalas cada vez menores, permitindo o desenvolvimento dos ramos da nanociência e da nanotecnologia. As propriedades físicas de estruturas em escala nanométrica (nanoestruturas) possibilitam a aplicação em ímãs permanentes, semicondutores, dispositivos magnéticos de armazenamento, entre outros. As nanoestruturas podem ser melhor compreendidas ao realizar-se estudos de suas propriedades físicas e as relações existentes entre as características estruturais e estas propriedades. O estudo de propriedades magnéticas é 
especialmente importante para a compreensão do magnetismo dos materiais como um todo e o domínio intelectual deste tópico permite elaborar novas aplicações e novos conceitos [2].

As nanoestruturas magnéticas têm se mostrado promissoras no desenvolvimento tecnológico por formarem domínios magnéticos, além de apresentarem propriedades magnéticas, elétricas, ópticas e químicas importantes, além de poder ter seu comportamento manipulado quando submetidos à variação de campo magnético externo. Um exemplo disso é a possibilidade de ganharem energia se submetidas à variação magnética em função do tempo, gerando aquecimento das nanoestruturas e tornando seu uso adequado como agentes epitérmicos e radioterápicos [1].

Há diversas geometrias possíveis de nanoestruturas magnéticas, dentre elas a geometria cilíndrica. Alguns trabalhos publicados têm mostrado a opção de inserir um furo no nanocilindro, modificando formação do estado de vórtice [3]. A esse tipo de estrutura dá-se o nome de nanoaneis magnéticos. Esses anéis têm espessura menor do que seu diâmetro e são geralmente depositados em substratos não-magnéticos. Este tipo de estrutura tem propriedades magnéticas estáticas e dinâmicas interessantes e sua investigação é importante para o desenvolvimento das tecnologias para criação de dispositivos de gravação magnética, podendo ser empregados como unidades de gravação, onde cada um armazena um bit [4].

Há diversos trabalhos realizados com nanoaneis com diferentes objetivos. Um dos interesses é o conhecimento da resposta ao campo magnético pela obtenção da curva de magnetização destes elementos. $\mathrm{O}$ trabalho de Lopez-Diaz mostra curvas de magnetização de anéis de NiFe de $1 \mu$ m de diâmetro externo [5]. Além disso, as configurações magnéticas destes anéis foram obtidas, mostrando a formação de vórtices em anéis e a dependência entre o campo necessário para aniquilação do vórtice e a espessura do nanoanel. O estudo dessas configurações magnéticas, isto é, a orientação dos momentos magnéticos ao longo da curva de histerese, é importante porque possibilita a descoberta de novos estados magnéticos e a identificação da correspondência com estados já conhecidos [6].

O trabalho de Muratov e Asitov propõe um modelo teórico de como nanoaneis ferromagnéticos de filmes finos podem ser utilizados como uma célula de memória magnética, podendo esta ser utilizada para armazenar, gravar e ler informações. O bit de informação é representado pela polaridade de uma parede de domínio estável de $360^{\circ}$. A transição entre dois estados magnéticos é feita pela aplicação de uma corrente em um fio que passa pelo anel, onde a parede de domínio de $360^{\circ}$ se divide em duas paredes de $180^{\circ}$ para então se moverem para a extremidade oposta e se recombinarem em uma parede de $360^{\circ}$ de polaridade oposta [7].

Há estudos que investigam as propriedades magnéticas e o processo de reversão de magnetização de nanoaneis isolados assimétricos, como função de defeitos geométricos. $\mathrm{O}$ trabalho de Palma tem como foco o comportamento do campo coercivo e da magnetização remanescente como função do tamanho de assimetrias presentes nos nanoaneis e também como função do ângulo de aplicação do campo externo, porém com o tamanho da amostra diferente do presente trabalho [8]. O trabalho de Ye estuda as propriedades magnéticas dinâmicas de nanoaneis de cobalto com defeitos inseridos em formato circular. Os resultados obtidos indicam que as curvas de histerese dos nanoaneis com defeitos pequenos são muito similares àquelas de anéis simétricos. Porém, quando a área do defeito aumenta, o processo de magnetização se torna diferente daquele exibido pelo nanoanel simétrico [9].

Considerando a possibilidade de nanoaneis magnéticos constituirem células de memória de alta performance, deve-se levar em consideração que nanoaneis defeituosos são inevitáveis em uma produção em massa. Os defeitos existentes podem afetar as propriedades magnéticas da nanoestrutura e consequentemente das células de memória em que serão utilizadas. Portanto, é importante estudar as propriedades de nanoaneis defeituosos na teoria. Neste trabalho serão estudadas as curvas de magnetização, bem como os estados magnéticos no ponto de remanência de nanoaneis defeituosos (sendo estes defeitos obtidos a partir de cortes na nanoestrutura de forma a deixa-la assimétrica), analisando o comportamento magnético como função da geometria e obtendo valores de remanência e coercividade de acordo com a geometria.

\section{REFERENCIAL TEÓRICO}

\subsection{Introdução ao nanomagnetismo}

Há registros acerca de fenômenos magnéticos desde a antiguidade, sendo que o conhecimento obtido nesta época, ainda não ditado pelo método científico, foi dominado pelos filósofos, e foi caracterizado por ter como bases superstições; este tipo de concepção perdurou até a renascença. O primeiro tratado, "De Magnete", publicado em 1600, foi escrito por William Gilbert. Foi o primeiro a considerar a Terra como um grande ímã e, 
além disso, reuniu os conhecimentos acumulados na época sobre a repulsão dos ímãs [10]. Com o surgimento do método científico e a descrição dos fenômenos pela análise matemática, com Galileu, Newton e outros no século XVII, e com o desenvolvimento da teoria coloumbiana da eletricidade, abriu-se espaço para o desenvolvimento da eletrodinâmica com Oersted, através análises dos efeitos magnéticos da corrente elétrica; com Biot e Savart, que descobriram a lei que permite determinar a intensidade do campo magnético gerado por uma corrente que flui em um condutor; com Arago, que realizou experiências acerca dos fenômenos observados por Oersted; e com Ampère, com a descoberta da força entre condutores paralelos, do comportamento de uma bobina circular como imã; entre outras contribuições [11].

As contribuições experimentais de Faraday, e teóricas de Maxwell foram um marco no final do século XIX. Faraday fez diversas contribuições, sendo as mais relevantes delas a lei da indução e a descrição de diversos materiais de acordo com o comportamento magnético. Maxwell foi o responsável por fornecer toda a base teórica da eletrodinâmica, por meio da formulação de suas equações. O século XX foi marcado pelo surgimento da mecânica quântica, possibilitando o entendimento moderno do magnetismo [12]. Fenômenos magnéticos de escala atômica foram descobertos na primeira metade do século passado e somente nas décadas mais recentes ficou claro que o magnetismo de estado sólido é, em grande parte, um fenômeno nanoestrutural [13].

O nanomagnetismo é o ramo da física que estuda propriedades magnéticas dos objetos na escala nanoscópica. Materiais que possuem partículas, filmes e outras estruturas nessa escala são definidos como materiais nanoestruturados. O nanomagnetismo tem ampla variedade de aplicações práticas, desde a geologia à gravação magnética, dos ferrofluidos até o transporte de medicamentos que podem ser direcionados a órgãos ou tecidos específicos. Porém, a aplicação mais bem sucedida deste ramo de estudo tem sido a gravação magnética, que tem proporcionado grande evolução na tecnologia da informação nas últimas cinco décadas [12].

A descoberta da magnetorresistência gigante foi um dos grandes estímulos para o desenvolvimento das aplicações do nanomagnetismo. A partir deste fenômeno pode-se desenvolver dispositivos como sensores magnetorresistivos e memórias magnetorresistivas de acesso aleatório (MRAM's). O armazenamento de dados digitais em MRAM's é mantido pelo momento magnético de nanoelementos em remanência, seja pela aplicação de um campo magnético ou pela passagem de uma corrente polarizada de spin. Dessa forma, para estas memórias funcionarem, mantendo a informação, não é necessário fornecer energia constantemente, ou seja, as MRAMs não são voláteis [6]. À medida em que a densidade de circuitos eletrônicos em chips dobra a cada dezoito meses (tendência estabelecida pela lei de Moore), a densidade de gravação magnética nos discos rígidos disponíveis no mercado duplica a cada dois meses [4]. A necessidade de aumentar a densidade de gravação em dispositivos de armazenamento de dados torna o estudo e desenvolvimento de nanoestruturas magnéticas para fins de gravação magnética um tema de pesquisa importante [6].

\subsection{Ciclo de histerese}

Os sistemas magnéticos são caracterizados pela forma como respondem à aplicação de um campo magnético externo. A suscetibilidade magnética $(\chi)$ mensura o comportamento dos materiais e diz respeito à forma como um material se magnetiza se for submetido a campo externo [14]. Existem diversos tipos de comportamento magnético, sendo os mais conhecidos o diamagnetismo, paramagnetismo, ferromagnetismo, ferrimagnetismo e antiferromagnetismo. Existem outros comportamentos, como metamagnetismo, superparamagnetismo, vidro de spin, speromagnetismo e helimangetico [12]. Neste trabalho, utiliza-se apenas o ferro como material, que é ferromagnético, pois materiais com este tipo de comportamento costumam ser utilizados em dispositivos lógicos nanomagnéticos. A relação entre a magnetização resultante e o campo aplicado em um material ferromagnético não é linear e a susceptibilidade é variável. A principal característica dessa classe de materiais é a capacidade de reter magnetização na retirada do campo externo [14].

A forma como uma amostra de material ferromagnético se comporta no processo de magnetização é expressa por um ciclo de histerese magnética, que mostra a magnetização em função do campo externo aplicado [14]. A diferença ou a largura da magnetização depende de diversos fatores como os parâmetros magnéticos do material, o formato, o acoplamento e a interação com outros elementos [15]. Três pontos importantes merecem destaque no ciclo de histerese:

- Saturação: dá-se o nome de magnetização de saturação ao valor da magnetização decorrente do alinhamento total dos domínios magnéticos pela aplicação de um campo externo suficientemente intenso. 
- Remanência: dá-se o nome de magnetização remanescente ao valor da magnetização quando o campo externo é totalmente removido partindo da saturação.

- Coercividade: refere-se à magnitude do campo necessário (campo coercivo) para reverter a magnetização, isto é, o ponto em que o ciclo de histerese cruza o eixo horizontal e a magnetização resultante é anulada.

A Figura 1 é exemplo de um ciclo de histerese de um material ferromagnético, mostrando os pontos importantes do ciclo: o campo coercivo indicado por $\mathrm{H}_{\mathrm{c}}$, a magnetização remanescente, indicada por $\mathrm{M}_{\mathrm{r}}$ e a magnetização de saturação, indicada por $\mathrm{M}_{\mathrm{s}}$.

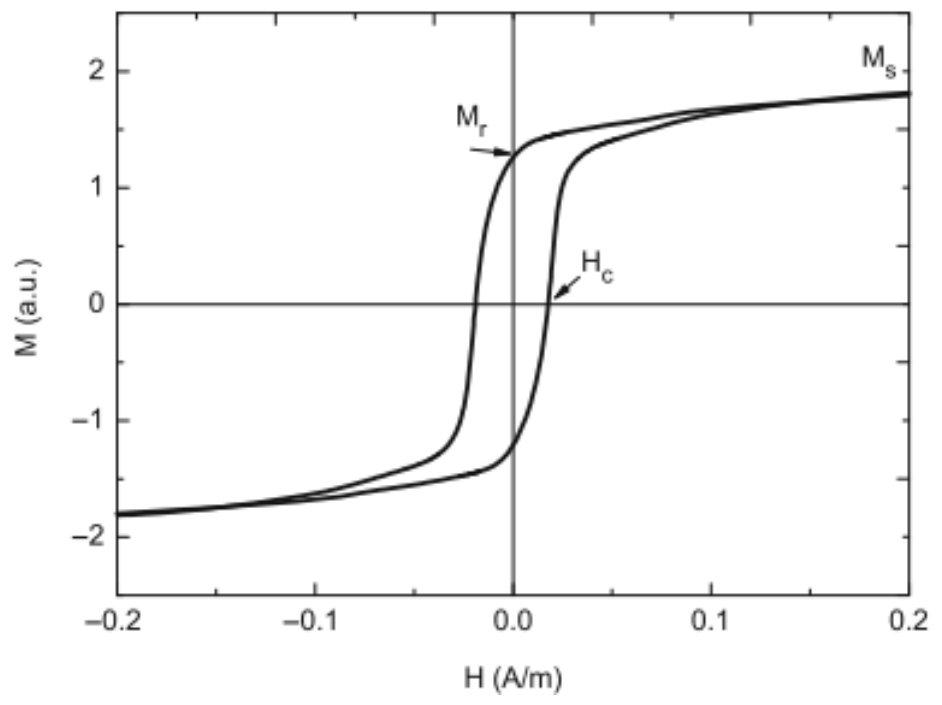

FIGURA 1. Histerese magnética típica de um material ferromagnético [16].

\subsection{Célula de simulação}

O ferro é um material que, devido à alotropia, pode apresentar mais de um tipo de estrutura, sendo que até a temperatura de $1200 \mathrm{~K}$, a estrutura é do tipo cúbica de corpo centrado [17]. A quantidade de átomos presentes numa amostra cúbica de material de lado d é dado pela Equação 1, onde $\mathrm{a}_{0}$ representa o parâmetro de rede. A fração representa a quantidade de células unitárias e n é a quantidade de átomos por célula. Para a estrutura cúbica de corpo centrado, a quantidade de átomos por célula é 2.

$$
\mathrm{N}=\mathrm{n}\left(\frac{\mathrm{d}}{\mathrm{a}_{0}}\right)^{3}
$$

Sabendo que o parâmetro de rede do ferro é $\mathrm{a}_{0}=0,297 \mathrm{~nm}$, o número de átomos presente numa amostra cúbica de lado $10 \mathrm{~nm}$ é de 84603 átomos, o que torna o cálculo das configurações magnéticas para todos os átomos inviável, quando utiliza-se amostras de dezenas ou centenas de nanômetros. Para contornar este problema, utiliza-se o conceito de célula de simulação. A célula de simulação representa um volume de um determinado material no qual os momentos magnéticos não variam seu sentido significativamente. O que determina o tamanho que essa célula deve ter é o comprimento de troca do material. Para tamanhos menores do que o comprimento de troca ltroca, a interação de troca é muito mais significativa do que a interação dipolar [14]. O comprimento de troca é dado pela Equação 2, onde Ms é a saturação e A é a rigidez de troca do material.

$$
1_{\text {troca }}=\sqrt{\frac{2 \mathrm{~A}}{\mu_{0} \mathrm{M}_{\mathrm{s}}^{2}}}
$$

\subsection{Energias magnéticas}

Para um sistema de i átomos e volume V, com momento magnético representado por $\overrightarrow{\mathrm{S}_{\mathrm{i}}}$, a energia total do sistema é dada pela Equação 3. Cada um dos termos envolvidos representa uma energia. 


$$
E_{\text {total }}=-J_{e} \sum_{j, i} \overrightarrow{S_{i}} \cdot \overrightarrow{S_{j}}-\vec{H} V M_{s} \sum_{i} \overrightarrow{S_{i}}-K V \sum_{i}\left(\overrightarrow{S_{i}}\right)^{2}+\frac{M_{s}^{2} V^{2}}{2} \sum_{i} \sum_{k} \frac{\overrightarrow{S_{i}} \cdot \overrightarrow{\mathrm{S}_{k}}}{r_{i k}^{3}}-\frac{3\left(\overrightarrow{S_{i}} \cdot \overrightarrow{r_{i k}}\right)\left(\overrightarrow{\mathrm{S}_{k}} \cdot \overrightarrow{r_{i k}}\right)}{r_{i k}^{5}}
$$

O primeiro termo representa a energia de troca, dada pela Equação 4, onde $\mathrm{J}_{\mathrm{e}}$ é a integral de troca, que pode ser positiva ou negativa. Caso seja positiva, o alinhamento é paralelo, caso contrário, é antiparalelo [18].

$$
\mathrm{E}_{\text {troca }}=-\mathrm{J}_{\mathrm{e}} \sum_{\mathrm{j}, \mathrm{i}} \overrightarrow{\mathrm{S}_{\mathrm{i}}} \cdot \overrightarrow{\mathrm{S}_{\mathrm{j}}}
$$

O segundo termo representa a energia Zeeman, dada pela Equação 5, onde $\overrightarrow{\mathrm{H}}$ é o campo externo, V é o volume da estrutura e $\mathrm{M}_{\mathrm{s}}$ é a magnetização de saturação do material.

$$
\mathrm{E}_{\text {zeeman }}=-\overrightarrow{\mathrm{H}} \mathrm{VM}_{\mathrm{s}} \sum_{\mathrm{i}} \overrightarrow{\mathrm{S}_{\mathrm{i}}}
$$

O terceiro termo representa a energia de anisotropia, dada pela Equação 6. Em função do ângulo, a energia de anisotropia uniaxial é dada pela Equação 7, onde K é a constante de anisotropia do material.

$$
\begin{gathered}
\mathrm{E}_{\text {anisotropia }}=-\mathrm{KV} \sum_{\mathrm{i}}\left(\overrightarrow{\mathrm{S}_{\mathrm{i}}}\right)^{2} \\
\mathrm{E}_{\text {anisotropia }}=\mathrm{KV} \operatorname{sen}^{2} \theta
\end{gathered}
$$

O quarto termo representa e energia dipolar, dada pela Equação 8.

$$
E_{\text {dipolar }}=\frac{\mathrm{M}_{\mathrm{s}}{ }^{2} \mathrm{~V}^{2}}{2} \sum_{\mathrm{i}} \sum_{\mathrm{k}} \frac{\overrightarrow{\mathrm{S}_{\mathrm{i}}} \cdot \overrightarrow{\mathrm{S}_{\mathrm{k}}}}{\mathrm{r}_{\mathrm{ik}}^{3}}-\frac{3\left(\overrightarrow{\mathrm{S}_{\mathrm{i}}} \cdot \overrightarrow{\mathrm{r}_{\mathrm{ik}}}\right)\left(\overrightarrow{\mathrm{S}_{\mathrm{k}}} \cdot \overrightarrow{\mathrm{r}_{\mathrm{ik}}}\right)}{\mathrm{r}_{\mathrm{ik}}^{5}}
$$

A seguir são descritas cada uma dessas energias por célula de simulação. Os campos locais são obtidos pelo divergente da energia de cada célula de simulação em relação ao seu momento.

\subsubsection{Energia de troca por célula de simulação}

A energia de troca tem origem na interação dos momentos magnéticos com seus primeiros vizinhos, sendo responsável pela tendência de alinhamento entre os momentos. Considerando a célula de simulação de lado d, a energia de troca de cada célula $\mathrm{i}$ atuando sobre seu vizinho j é dada pela Equação 9 , onde $\overrightarrow{\mathrm{m}_{\mathrm{i}}}$ e $\overrightarrow{\mathrm{m}_{\mathrm{j}}}$ são os momentos magnéticos das células vizinhas. O campo de troca produzido por um vizinho j na célula i é dado pela Equação 10

$$
\begin{gathered}
\frac{E_{\text {troca }}}{\mathrm{d}^{3}}=\frac{\mathrm{A}}{\mathrm{d}^{2}} \sum_{\mathrm{j}, \mathrm{i}}\left(1-\mathrm{m}_{\mathrm{i}} \cdot \overrightarrow{\mathrm{m}_{\mathrm{j}}}\right) \\
\overrightarrow{\mathrm{H}}_{\text {troca }}=\frac{\mathrm{A}}{\mathrm{M}_{\mathrm{s}} \mathrm{d}^{2}} \sum_{\mathrm{j}} \overrightarrow{\mathrm{m}_{\mathrm{j}}}
\end{gathered}
$$

\subsubsection{Energia Zeeman por célula de simulação}

A energia Zeeman tem origem na interação dos momentos magnéticos com o campo magnético externo aplicado. Na aplicação de um campo externo, um torque é realizado de modo a fazer o momento se alinhar com o campo aplicado. A energia Zeeman é minimizada quando o momento e o campo externo estão alinhados. Para a célula de simulação de lado d, a energia Zeeman de uma célula i é dada pela Equação 11. Calculando o campo pelo divergente da energia, o resultado é que o campo Zeeman é igual ao campo externo aplicado para todas as células de simulação, conforme a Equação 12. 


$$
\begin{gathered}
\frac{E_{\text {zeeman }}}{\mathrm{d}^{3}}=-\overrightarrow{\mathrm{H}} \mathrm{M}_{\mathrm{s}} \sum_{\mathrm{i}} \overrightarrow{\mathrm{m}_{\mathrm{i}}} \\
\overrightarrow{\mathrm{H}}_{\text {zeeman }}=\overrightarrow{\mathrm{H}}
\end{gathered}
$$

\subsubsection{Energia de anisotropia por célula de simulação}

A energia de anisotropia está associada ao fato de a magnetização de um material tender a ter uma direção preferencial. Considerando a célula de simulação de lado d, a energia de anisotropia, em função do ângulo de entre a magnetização e o eixo fácil, é dada pela Equação 13, onde K é a constante de anisotropia do material. Neste trabalho, foi considerado o tipo de anisotropia mais simples, isto é, a uniaxial, em que existe apenas uma direção preferencial, isto é, o eixo fácil de magnetização. O campo de anisotropia sobre cada célula é dado pela Equação 14

$$
\begin{aligned}
& \frac{E_{\text {anisotropia }}}{\mathrm{d}^{3}}=\mathrm{Ksen}^{2} \theta \\
& \overrightarrow{\mathrm{H}}_{\text {anisotropia }}=\frac{2 \mathrm{~K}}{\mathrm{M}_{\mathrm{s}}} \overrightarrow{\mathrm{m}_{\mathrm{i}}}
\end{aligned}
$$

\subsubsection{Energia dipolar por célula de simulação}

A energia dipolar está associada às interações entre os momentos magnéticos um a um, ou seja, leva em conta a influência que cada um tem em todos os outros. É uma interação de longo alcance. Para a célula de simulação de lado d, a energia dipolar é dada pela Equação 15 , onde o vetor $\overrightarrow{n_{\mathrm{ik}}}$ representa a distância entre as células de simulação i e $\mathrm{k}$.

$$
\frac{E_{\text {dipolar }}}{d^{3}}=\frac{M_{s}^{2}}{2} \sum_{i} \sum_{k} \frac{\overrightarrow{\mu_{i}} \cdot \overrightarrow{\mu_{k}}}{n_{i k}^{3}}-\frac{3\left(\overrightarrow{\mu_{i}} \cdot \overrightarrow{n_{i k}}\right)\left(\overrightarrow{\mu_{k}} \cdot \overrightarrow{n_{i k}}\right)}{n_{i k}^{5}}
$$

O campo dipolar é apontando na direção p, que representa as possíveis direções x, y e z, é dado dado pela Equação 16. A apresentação da Equação na forma generalizada se dá porque para cada direção, o campo dipolar terá uma expressão dada pela derivada parcial da energia dipolar em relação à direção referida.

$$
\overrightarrow{\mathrm{H}}_{\text {dipolar }}^{\mathrm{p}}=-\frac{1}{\mathrm{M}_{\mathrm{s}}} \frac{\partial \mathrm{E}_{\text {dipolar }}}{\partial \overrightarrow{\mathrm{m}_{\mathrm{i}}^{\mathrm{p}}}}
$$

\subsection{Configurações dos momentos magnéticos e dispositivos de memória com utilização de nanoaneis}

A Anéis magnéticos e discos de dimensões nanoscópicas ou mesoscópicas, têm propriedades físicas que podem ser usadas para codificar informações digitais. A configuração vórtice presentes nessas estrututras, por exemplo, têm potencial para armazenamento de dados. Nanodiscos e nanoaneis têm a vantagem de, uma vez que são estruturas com fechamento na configuração da magnetização, apresentar baixos campos de dispersão. Isso é importante para aplicações práticas, pois as nanoestruturas formadas com esses elementos podem ser arranjadas em ordenamentos mais compactos, tendo em vista a redução das interações entre vizinhos [16].

Os nanoaneis têm várias configurações de spin possíveis, com propriedades como o sentido de rotação do vórtice, a direção da magnetização no estado cebola ou no estado tipo ferradura que podem ser utilizadas para dados digitais. Estes estados estão ilustrados na Figura 2. Há também outras possibilidades de configurações, a depender das características da amostra, como o estado C, ilustrado na Figura 3. 


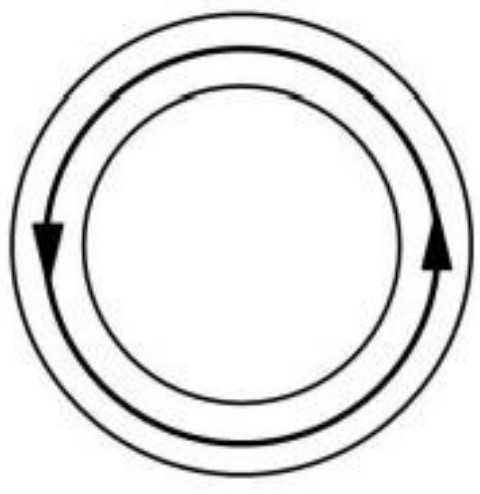

(a)

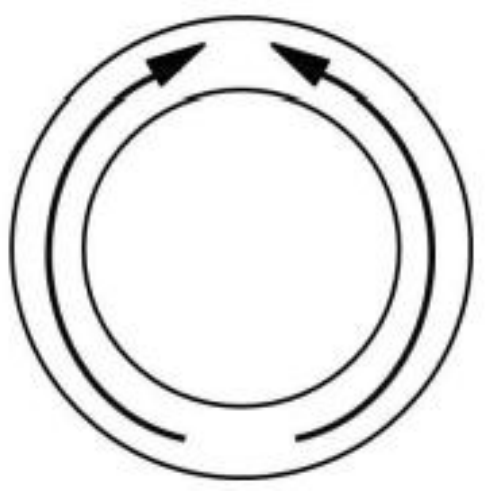

(b)

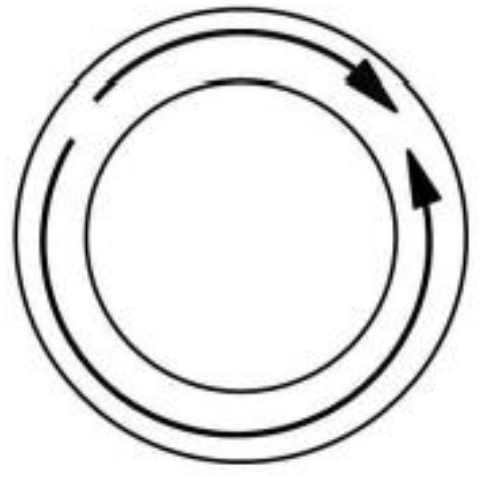

(c)

FIGURA 2. Configurações de spin para nanoaneis: (a) vórtice; (b) cebola; (c) ferradura [6].

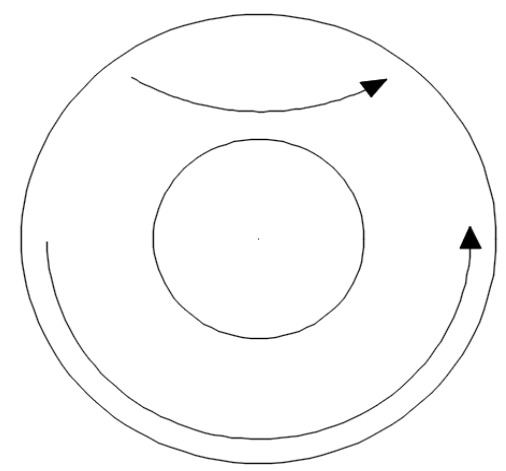

FIGURA 3. Configuração de spin do tipo C (Autoria própria).

Um arranjo simples de nanoaneis que pode ser usado para armazenamento de dados é uma estrutura de três camadas formada por dois nanoaneis magnéticos superpostos separados por uma camada não magnética, constituindo uma válvula de spin [16], conforme ilustrado pela Figura 4.

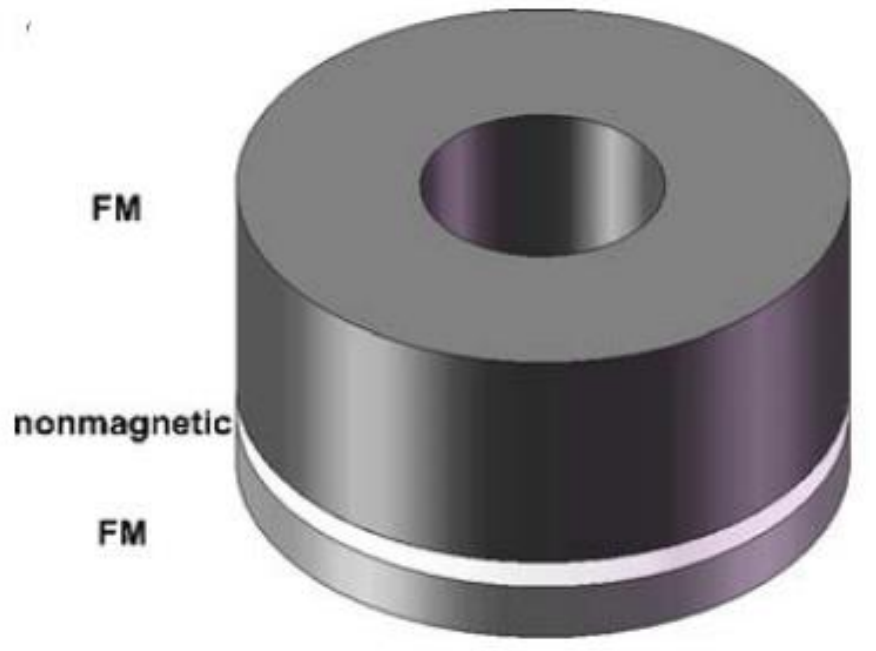

FIGURA 4. Célula de memória de nanoaneis magnéticos [16].

Essa estrutura de nanoaneis pode ter seu estado magnético controlado por uma corrente elétrica fluindo ao longo do comprimento. Usando o efeito da magnetorresistência gigante, a variação da resistência permite a identificação de momentos magnéticos paralelos ou antiparalelos nos nanoaneis ferromagnéticos, permitindo a leitura das informações registradas [16]. 


\section{Metodologia}

O método computacional utilizado para simular os resultados deste trabalho foi desenvolvido em linguagem de programação Fortran por pesquisadores da Universidade Federal do Rio Grande do Norte (UFRN) e posteriormente adaptado às necessidades deste trabalho. A análise do programa parte da Equação 17, conhecida como equação de Landau-Lifshitz-Gilbert. O objetivo é que todos os momentos dipolo magnéticos alinhem-se com o campo magnético médio local, dentro de uma determinada tolerância, de modo que o torque seja minimizado o máximo possível.

$$
\mathrm{d} \overrightarrow{\mathrm{M}} / \mathrm{dt}=-\gamma \overrightarrow{\mathrm{M}} \times \overrightarrow{\mathrm{H}}_{\text {local }}
$$

$\mathrm{Na}$ Equação 17, $\gamma$ representa a razão giromagnética. O campo local é obtido a partir de várias energias envolvidas. Há alguns parâmetros importantes a serem considerados para a determinação de cada energia envolvida no processo. Esses parâmetros variam de acordo com o material utilizado. Para o ferro, os parâmetros estão descritos na Tabela 1.

TABELA 1. Parâmetros magnéticos do ferro (Autoria própria).

\begin{tabular}{cc}
\hline Parâmetro & Magnitude \\
\hline $\mathrm{M}_{\mathrm{S}}(\mathrm{A} / \mathrm{m})$ & $1,7 \times 10^{6}$ \\
\hline $\mathrm{A}(\mathrm{J} / \mathrm{m})$ & $2,5 \times 10^{-11}$ \\
\hline $\mathrm{H}_{\mathrm{TROCA}}(\mathrm{T})=\mathrm{A} / \mathrm{M}_{\mathrm{S}} \mathrm{d}^{2}$ & $14,7 \times \mathrm{d}^{2}$ \\
\hline $\mathrm{K}\left(\mathrm{J} / \mathrm{m}^{3}\right)$ & $4,7 \times 10^{4}$ \\
\hline $\mathrm{H}_{\mathrm{ANIS}}=2 \mathrm{~K} / \mathrm{M}_{\mathrm{S}}\left(10^{4} \mathrm{G}\right)$ & 0,055 \\
\hline $\mathrm{L}_{\mathrm{TROCA}}=\pi \sqrt{\frac{2 \mathrm{~A}}{\mu_{0} \mathrm{M}_{\mathrm{S}}^{2}}}$ & $11,65 \mathrm{~nm}$ \\
\hline
\end{tabular}

Primeiramente, inicializa-se o sistema com uma dada configuração magnética e calcula-se o campo local que age sobre cada uma das células de simulação. O valor calculado do torque entre a direção do momento magnético e o campo médio da configuração magnética é comparado com uma dada tolerância praticamente nula para que o torque seja minimizado. Se o torque for menor que a tolerância previamente estabelecida, aceita-se a configuração e o sistema converge. Repete-se esta etapa até que o torque seja minimizado. Se o número máximo de repetições for ultrapassado, o sistema não converge e busca nova configuração. A Figura 5 mostra o fluxograma que descreve o funcionamento do programa.

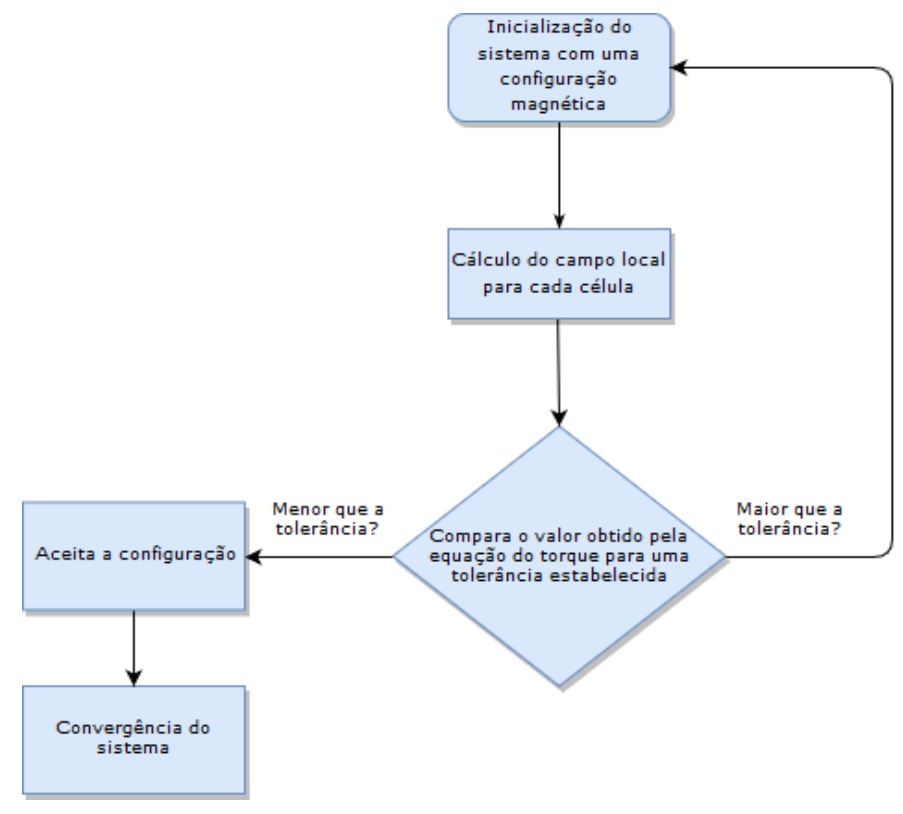

FIGURA 5. Fluxograma do método computacional (Autoria própria). 
Neste trabalho o tamanho da célula adotado foi de $3 \mathrm{~nm}$ de lado e o campo magnético externo foi aplicado na direção x. O diâmetro fixo das amostras foi de $51 \mathrm{~nm}$ e a espessura de $15 \mathrm{~nm}$. O trabalho concentrou-se em anéis definidos pela altura do corte $\mathrm{d}$, seu raio externo $\mathrm{R}$ e seu raio interno $\mathrm{r}$. São estabelecidos os parâmetros $\alpha$ e $\beta$, que são respectivamente definidos como as razões d/R e r/R. Quanto maior é o $\beta$, mais estreito é o anel. $O$ parâmetro $\alpha$ define o tamanho da assimetria, e um anel simétrico tem $\alpha=1,0$. A Figura 6 ilustra os parâmetros.

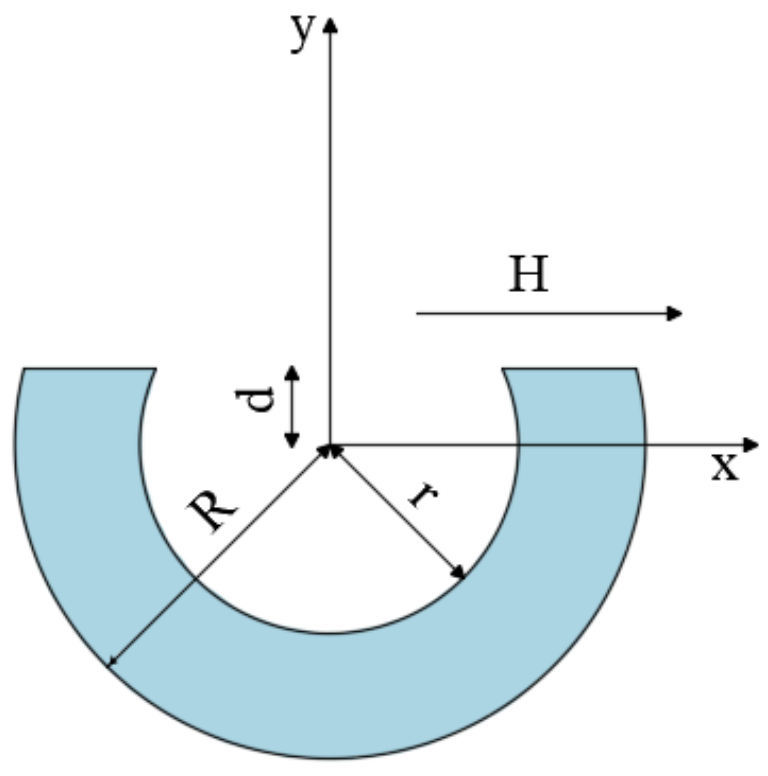

FIGURA 6. Parâmetros geométricos variáveis dos nanoaneis (Autoria própria).

\section{RESULTADOS E DISCUSSÕES}

Inicia-se a análise da influência dos defeitos no processo de reversão da magnetização. Os gráficos obtidos mostram as curvas de histerese para diferentes valores de $\alpha$. A Figura 7 mostra as curvas de histerese para $\beta=0,0$. Essa configuração corresponde a um disco. Para este caso, nota-se que para valores menores de $\alpha$, isto é, para anéis com defeitos cada vez maiores, o campo coercivo apresenta valores crescentes e a magnetização remanescente apresenta valores decresentes. Nota-se também que quanto mais simétrico é o anel, mais difícil torna-se saturá-lo. O cruzamento existente na curva para $\alpha=1,00$ sugere que o processo de reversão de magnetização ocorre por nucleação de um vórtice.

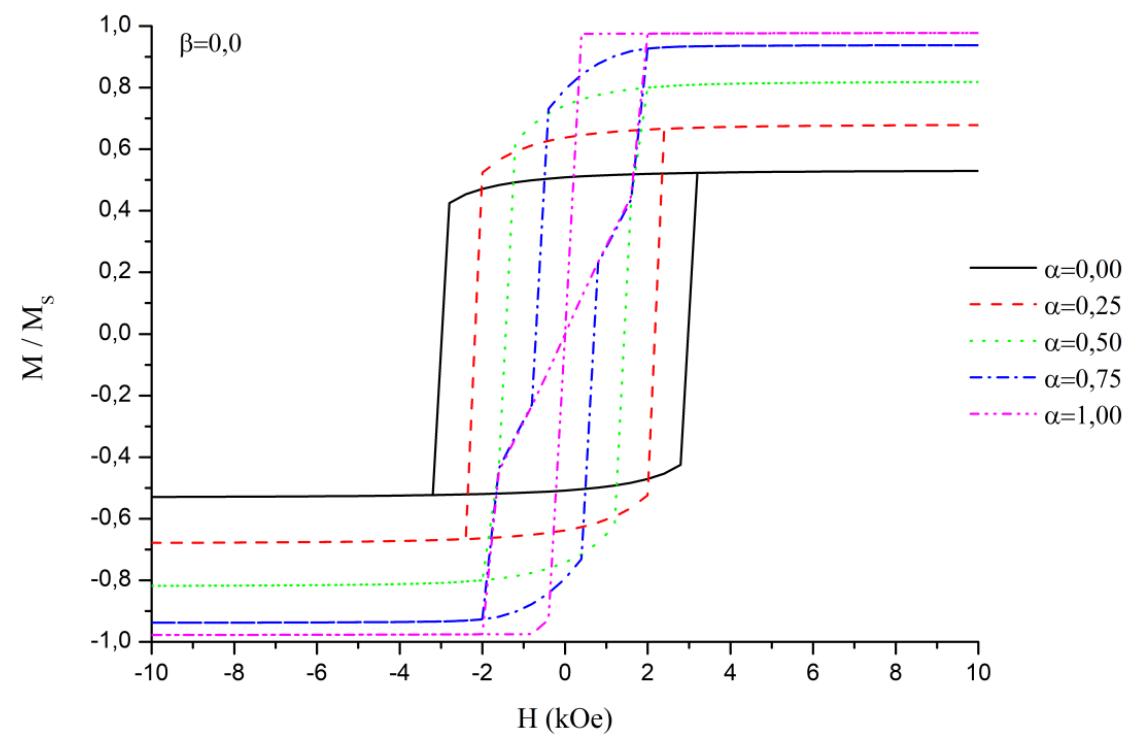

FIGURA 7. Curvas de histerese para $\beta=0,0$ (Autoria própria). 
As Figura 8, 9 e 10 mostram as curvas de histerese para $\beta=0,2 ; \beta=0,4$ e $\beta=0,6$ respectivamente. Percebe-se mudança considerável nos formatos das curvas em relação às curvas correspondentes a $\beta=0,0$. Para o caso da Figura 8 , nota-se semelhança com a curva anterior para $\alpha=0,0$, porém com campo necessário menor para saturar a amostra e menor campo coercivo. Nota-se novamente a influência do defeito na amostra, pois novamente o aumento dos defeitos (valores menores de $\alpha$ ) produz menor magnetização remanescente e campo coercivo maior. Nas Figura 9 e 10, a mudança nos formatos das curvas são maiores, isso porque apesar de o campo coercivo continuar se comportamento da mesma forma anterior, isto é, aumentando conforme o valor de $\alpha$ decresce, o comportamento da magnetização não segue o padrão anterior e tem um comportamento inconstante com a variação de $\alpha$.

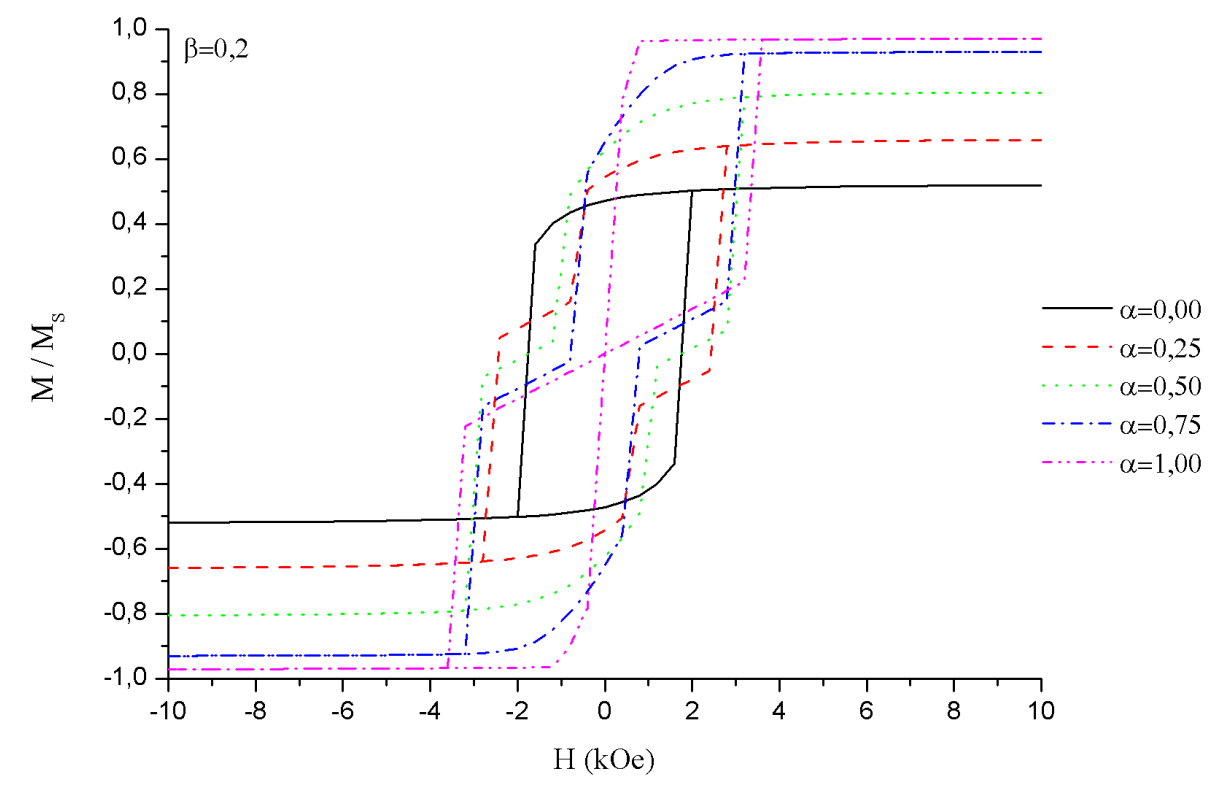

FIGURA 8. Curvas de histerese para $\beta=0,2$ (Autoria própria).

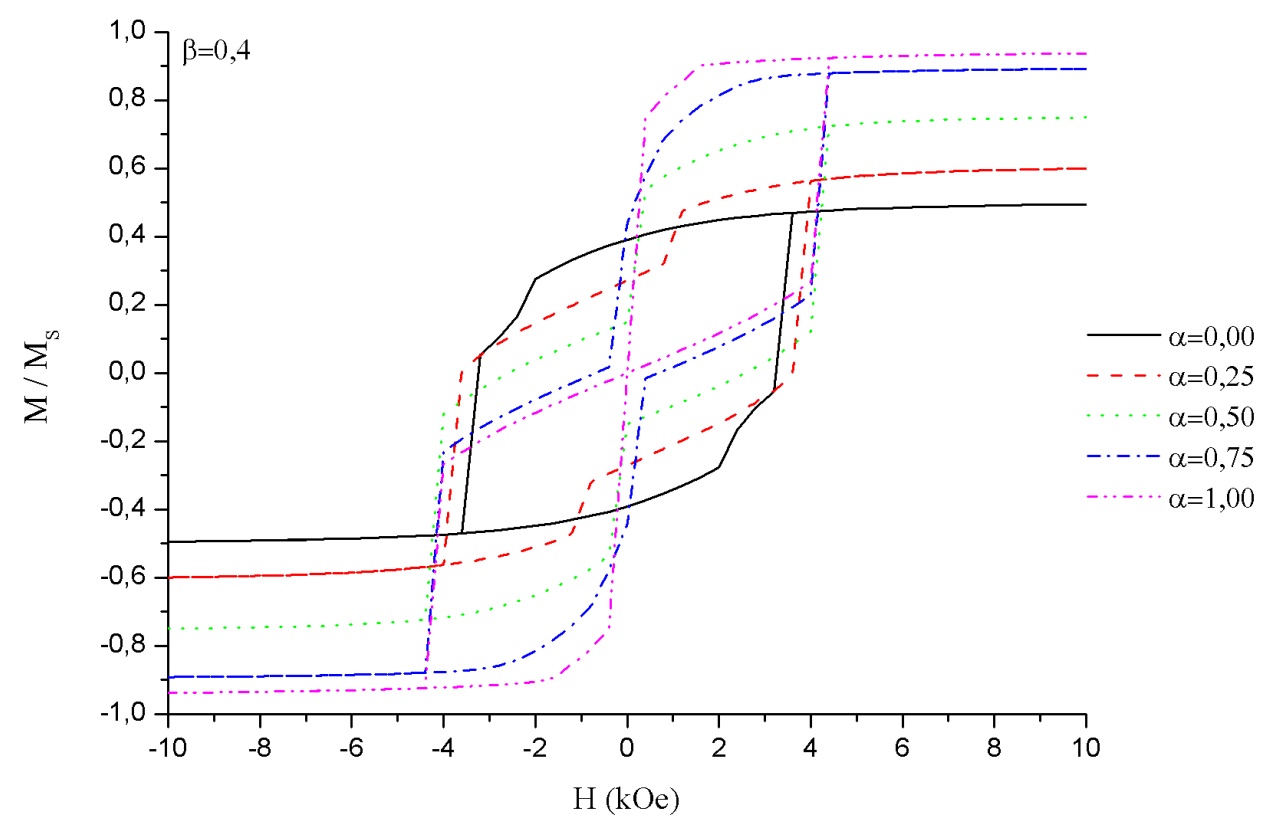

FIGURA 9. Curvas de histerese para $\beta=0,4$ (Autoria própria). 


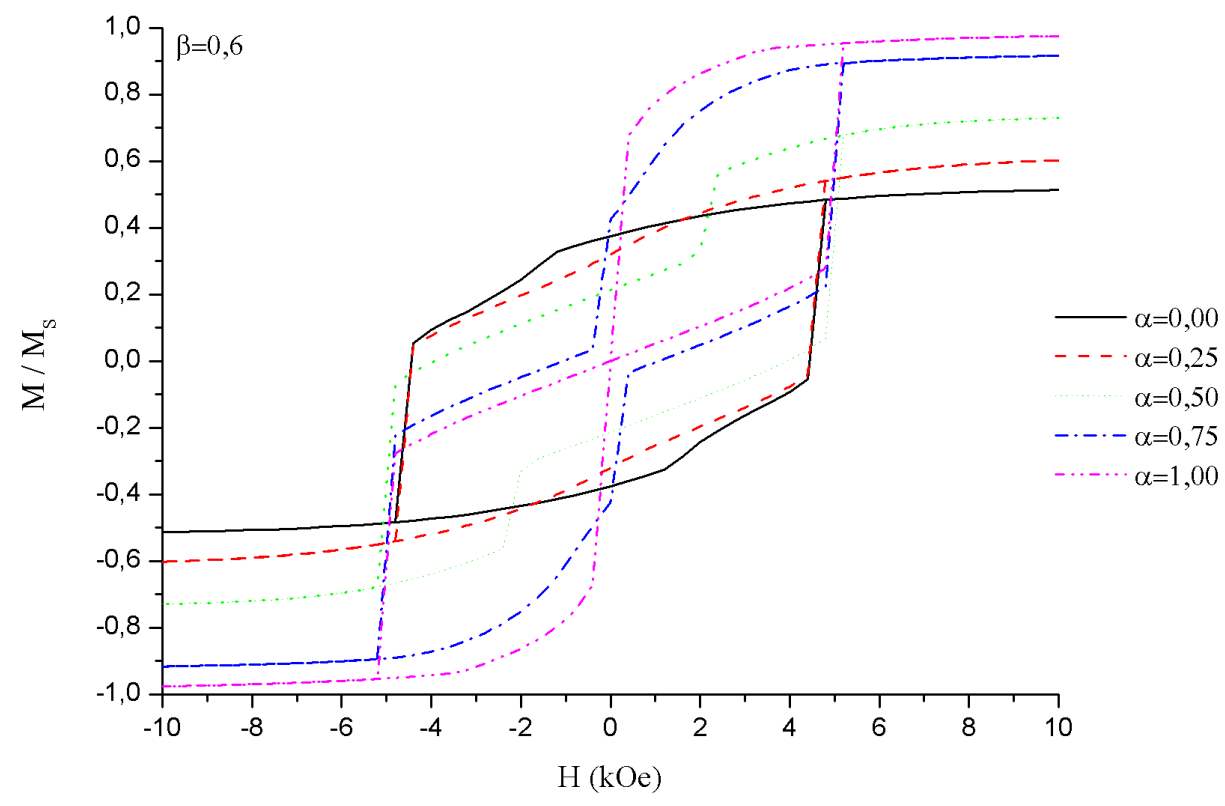

FIgURA 10. Curvas de histerese para $\beta=0,6$ (Autoria própria).

A Figura 11 mostram as curvas de histerese para $\beta=0,8$. Para a situação em que o anel é simétrico, como nos resultados anteriores, o campo coercivo e a magnetização remanescente são nulos, porém após a inserção dos defeitos e conforme aumenta-se os defeitos, a magnetização remanescente tende a crescer. O campo coercivo também apresenta comportamento crescente, como foi verificado nos resultados anteriores. Descobriu-se que, para a maioria dos anéis estudados, a diminuição da assimetria leva a uma necessidade de campo maior para atingir a saturação.

As Figuras 12 e 13 mostram os mapas de spins no processo de reversão de magnetização para o ponto em que o campo aplicado é anulado, para as curvas de $\beta=0,1$ a $\beta=0,8$, sendo o valor de $\alpha=0,75$ o mesmo para todos. A barra de cores referente ao plano da figura representa o ângulo dos momentos magnéticos no plano e a barra de cores referente às setas representa o ângulo fora do plano, ambos em graus. Em todos os casos das figuras, o processo de reversão de magnetização ocorre com o surgimento do estado C.

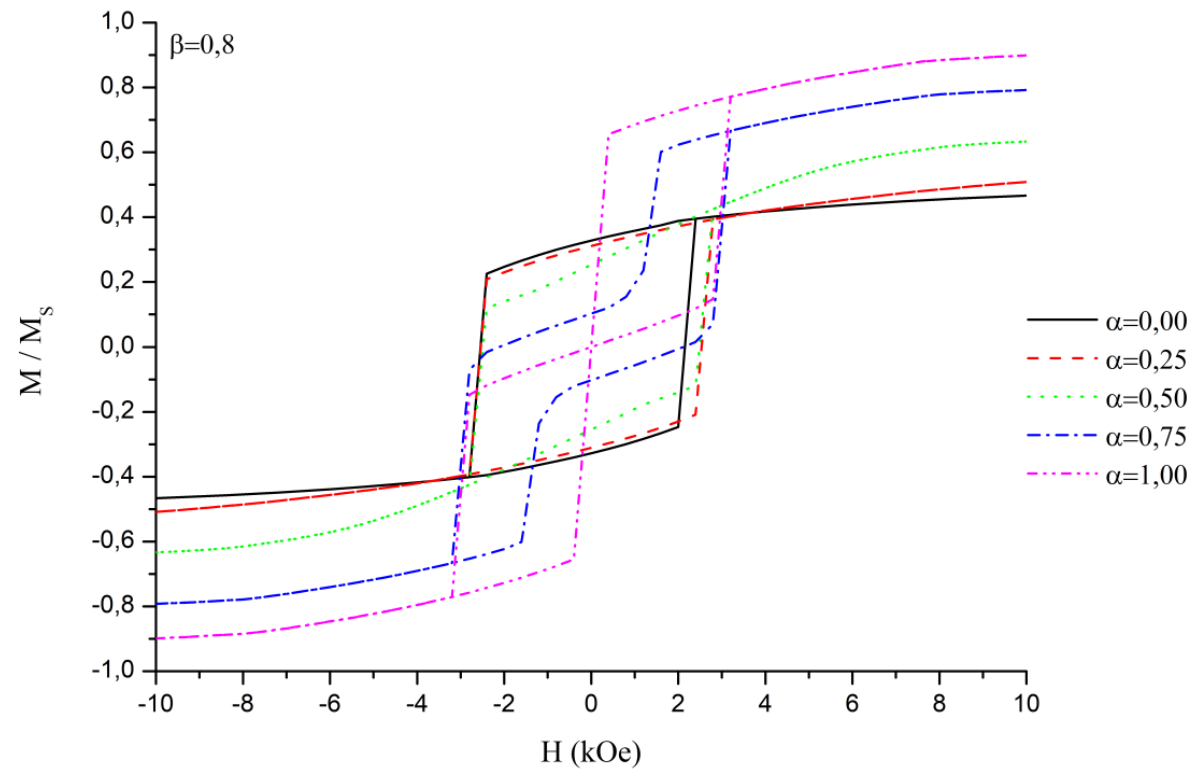

FIgURA 11. Curvas de histerese para $\beta=0,8$ (Autoria própria).

O estado apresentado nesses casos é fortemente influenciado pela presença dos defeitos. Quando as amostras são totalmente simétricas, todas as amostras apresentam vórtice na remanência. Quando aumenta-se o 
valor do raio interno, torna-se mais difícil que a amostra consiga tender a um único domínio, por conta da geometria, então, o aumento do valor de $\beta$ resulta no aumento da estabilidade do estado vórtice. Quando as amostras são assimétricas, a geometria impede o padrão de fechamento da magnetização, fazendo com que as amostram tendam a assumir o estado C. Observa-se pelas figuras que o crescimento do raio interno também influencia na estabilidade do estado $C$, de modo que para valores menos de $\beta$, há maior tendência de alinhamento. Portanto, para anéis simétricos, $\beta$ aumenta a estabilidade do vórtice e para anéis defeituosos, $\beta$ aumenta a estabilidade do estado C.

a)

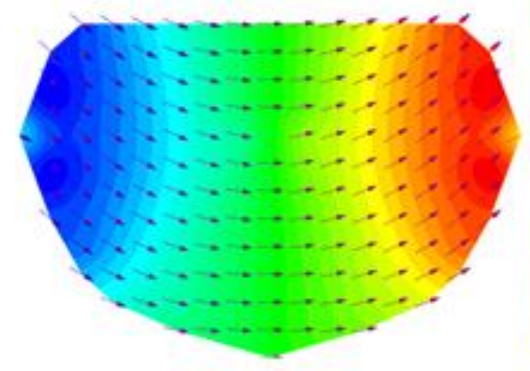

c)

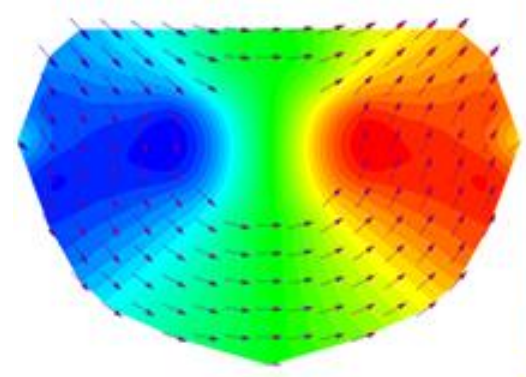

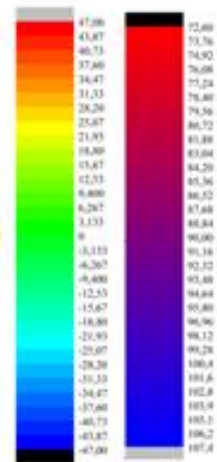

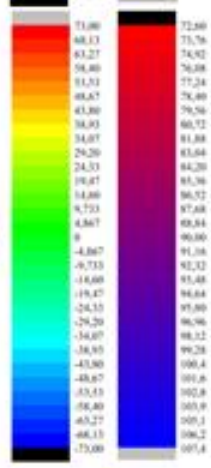

b)

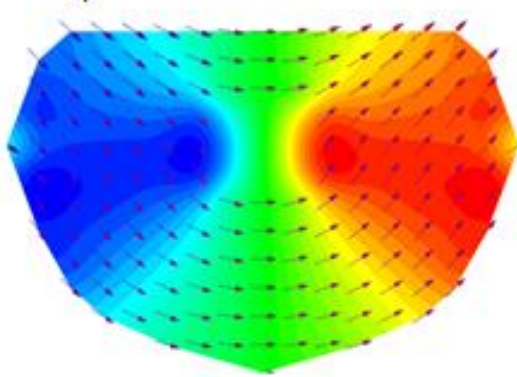

d)

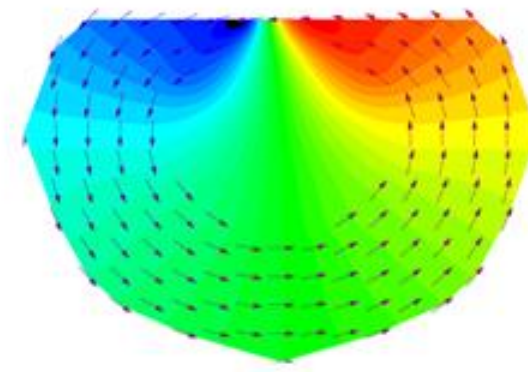

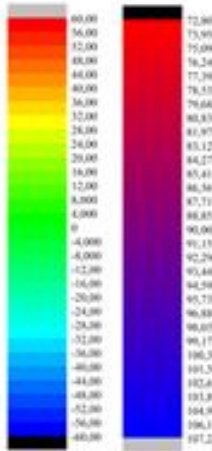

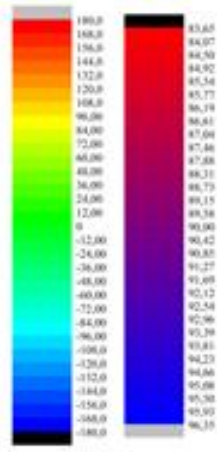

FigURA 12. Mapas de spins para: a) $\beta=0,1$; b) $\beta=0,2$; $) \beta=0,3$; d) $\beta=0,4$ (Autoria própria).

a)

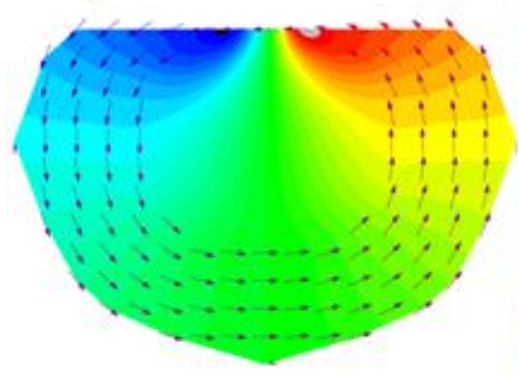

c)

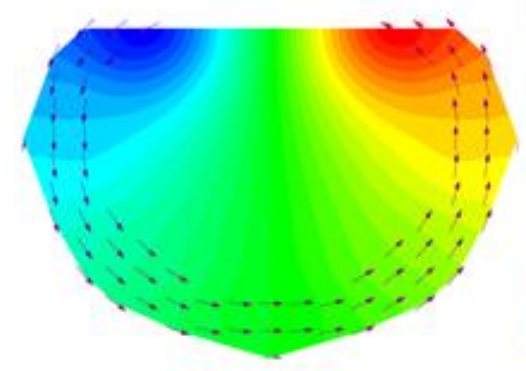

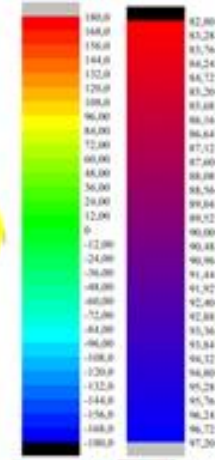

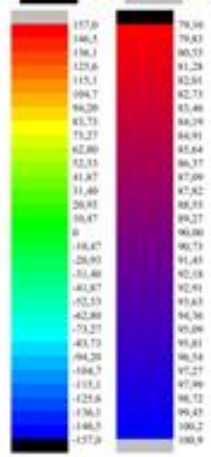

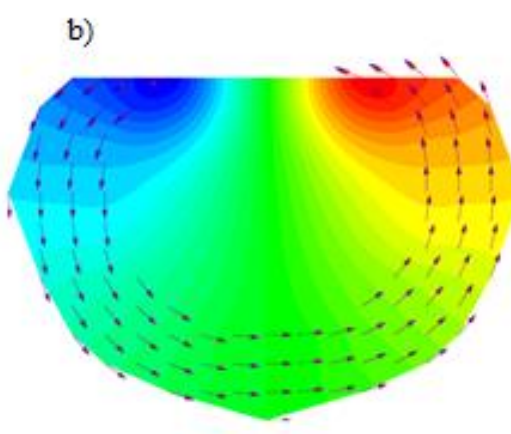

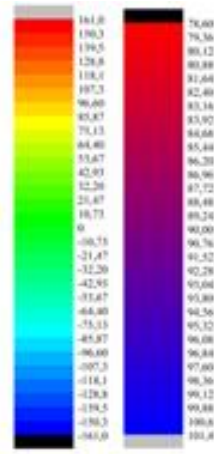

d)
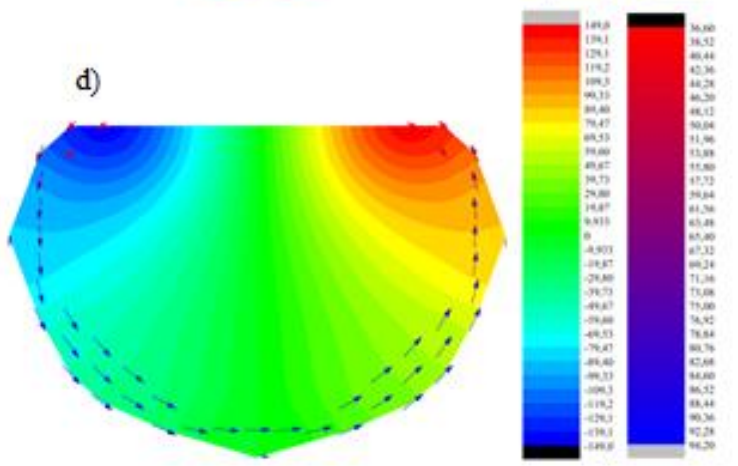

FIGURA 13. Mapas de spins para: a) $\beta=0,5$; b) $\beta=0,6$; c) $\beta=0,7$; d) $\beta=0,8$ (Autoria própria). 


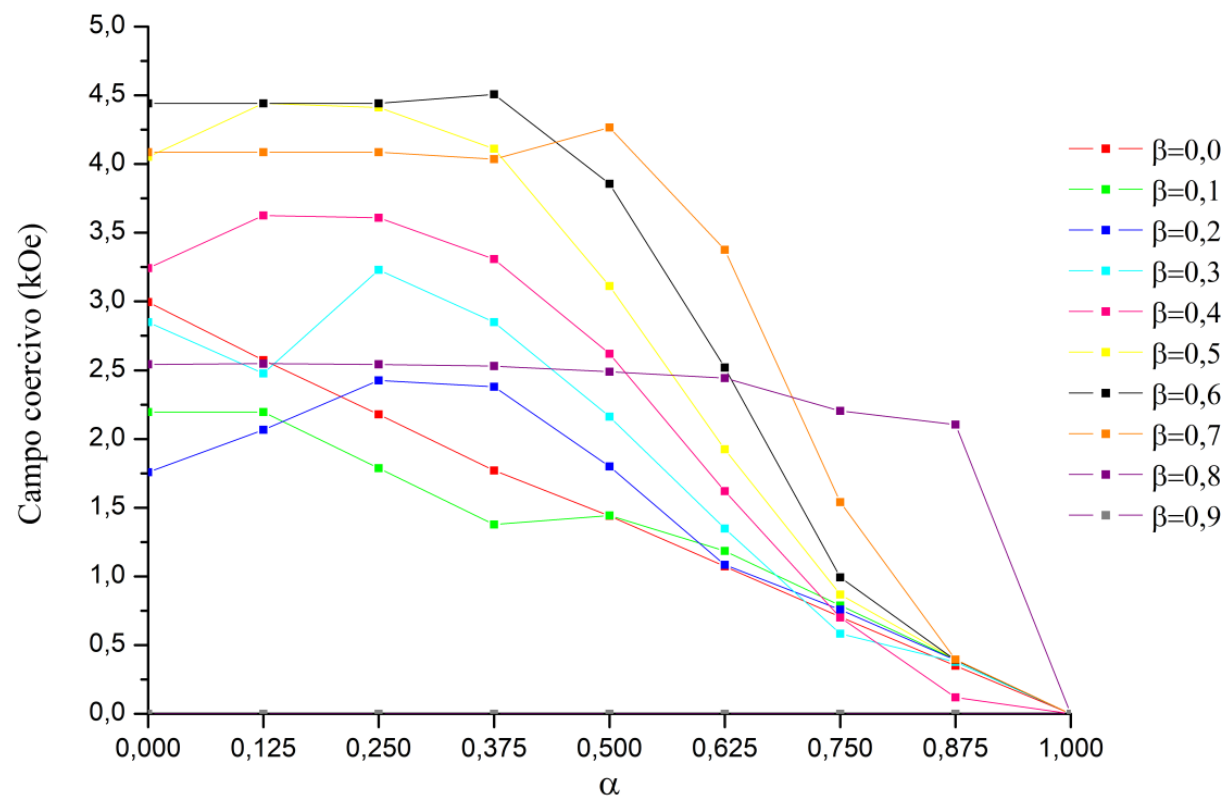

FIGURA 14. Campo coercivo para todos os valores de $\beta$ e $\alpha$ simulados (Autoria própria).

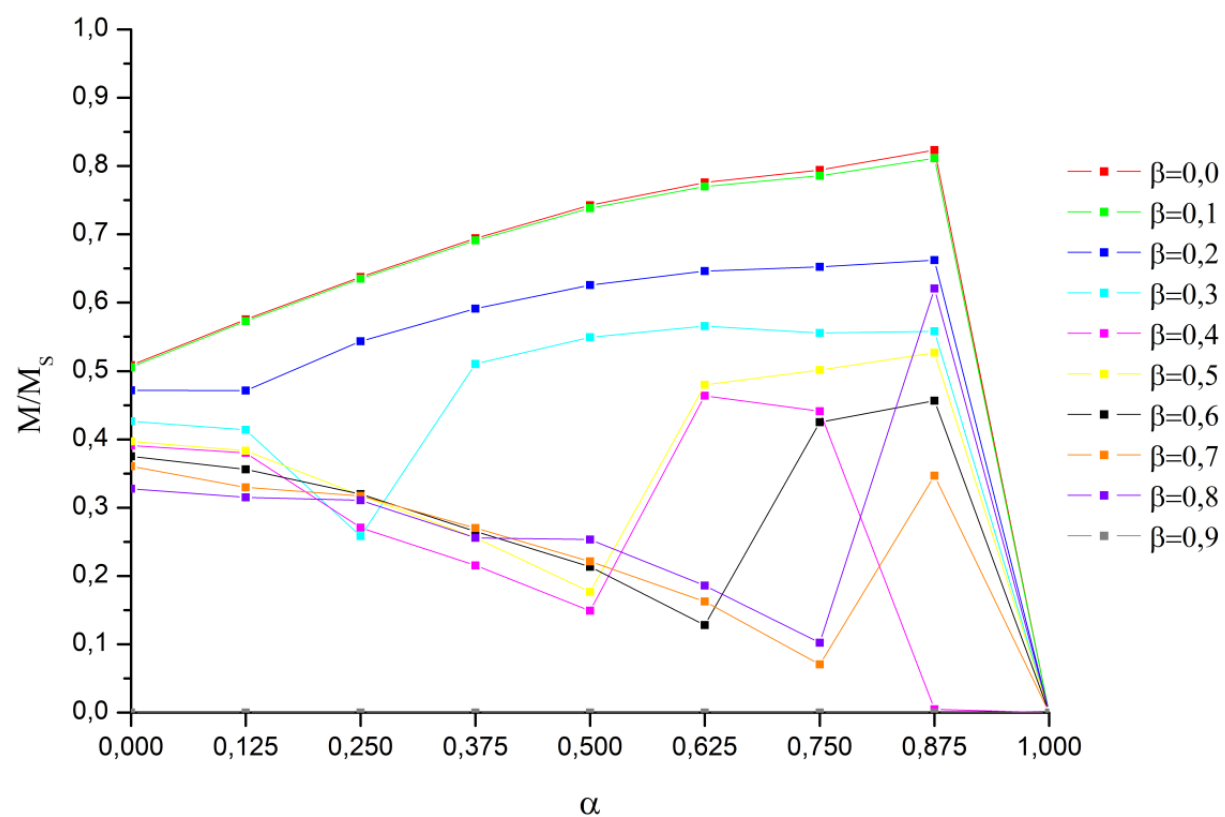

FIGURA 15. Valores de magnetização remanescente para todos os valores de $\beta$ e $\alpha$ simulados (Autoria própria).

\section{CONCLUSÃO}

Através deste trabalho foi possível analisar a influência de parâmetros geométricos em nanoaneis de ferro. Os resultados encontrados mostram que a inserção dos defeitos, tornando os nanoaneis assimétricos têm forte influência no processo de magnetização das amostras. Descobriu-se que de forma geral, o aumento da assimetria leva a um aumento do campo coercivo em todas as espessuras estudadas e que, para nanoaneis de raio interno menores, há uma tendência de aumento da magnetização remanescente, conforme diminui-se a assimetria, seguida de uma queda abrupta na magnetização para o caso de um anel simétrico. Para nanoaneis de raio interno maior, a magnetização remanescente não segue um comportamento padrão. Descobriu-se que, para a maioria dos anéis estudados, a diminuição da assimetria leva a uma necessidade de campo maior para atingir a saturação. Os resultados encontrados podem ser utilizados para trabalhos futuros que busquem o desenvolvimento de dispositivos baseados em spintrônica, já que é importante conhecer o comportamento das estruturas utilizadas. Outros trabalhos podem futuramente estudar os mesmos efeitos em nanoaneis de outros 
materiais. Além disso, outros trabalhos podem ser feitos com outros tipos de formato de defeitos inseridos, ampliando a base teórica para possíveis utilizações futuras dessas nanoestruturas.

\section{REFERÊNCIAS}

[1] QUEIROZ, Francisco Gomes de. Fases Magnéticas de Nanopartículas Esféricas tipo Core@Shell com Núcleo e Casca de Fe separados por Material não Magnético. 2017. 85 f. Dissertação (Mestrado) - Curso de Mestrado em Física, Universidade do Estado do Rio Grande do Norte, Mossoró, 2017.

[2] CAMPOS, Cecília Leite do Amaral Veras. Propriedades magnéticas de arranjos de nanofios de níquel eletrodepositados em membranas porosas de óxido de alumínio. 2016. 91 f. Tese (Doutorado) - Curso de Mestrado em Física, Universidade Federal de Pernambuco, Recife, 2016.

[3] DIAS, Carlos Sato Baraldi. Estudo de vórtice magnético em nanopartículas para aplicações em hipertermia magnética. 2014. 183 f. Tese (Doutorado) - Curso de Doutorado em Ciências, Universidade Estadual de Campinas, Campinas, 2014.

[4] GUIMARÃES, Alberto P.. Introdução ao nanomagnetismo. Rio de Janeiro: Centro Brasileiro de Pesquisas Físicas, 2006.

[5] LOPEZ-DIAZ, L, et al. Computational Study of First Magnetization Curves in Small Rings. IEEE Trans. Magn., v. 36, n. 5, p. 3155, 2000.

[6] PESSOA, Nathan Lima. Controle de paredes de domínio em nanoaneis de NiFe em estruturas núcleo-casca cilíndricas com acoplamento dipolar. 2017. 78 f. Dissertação (Mestrado) - Curso de Mestrado em Física, Universidade Federal do Rio Grande do Norte, Natal, 2017.

[7] MURATOV, C.b.; OSIPOV, V.v.. Bit Storage by $360^{\circ}$ Domain Walls in Ferromagnetic Nanorings. Ieee Transactions On Magnetics, [s.1.], v. 45, n. 8, p.3207-3209, ago. 2009.

[8] PALMA, J.l. et al. Micromagnetic simulation of Fe asymmetric nanorings. Journal Of Magnetism And Magnetic Materials, [s.1.], v. 324, n. 4, p.637-641, fev. 2012.

[9] YE, Qingying et al. Magnetic dynamic properties of defective cobalt nanorings: Monte Carlo simulation. Journal Of Magnetism And Magnetic Materials, [s.1.], v. 473, p.301-305, mar. 2019.

[10] GUIMARÃES, Alberto P.. Os 400 anos do De magnete. Disponível em: <http://www.cbpf.br/ labmag/apg.pdf>. Acesso em: 02 nov. 2018.

[11] BASSALO, José Maria Filardo. Eletrodinâmica Clássica. São Paulo: Livraria da Física, 2007.

[12] NOVAK, Miguel A.. Introdução ao magnetismo. In: ESCOLA BRASILEIRA DE MAGNETISMO, 2. 1999, Rio de Janeiro. Palestra. Rio de Janeiro: Sbf, 1999. p. 1 - 13.

[13] SKOMSKI, R. Nanomagnetics. Journal Of Physics: Condensed Matter, [s.1.], v. 15, n. 20, p.841-896, 9 maio 2003.

[14] MARTINS JUNIOR, Sergio Murilo da Silva Braga. Efeito do campo dipolar em Portas Lógicas Nanomagnéticas. 2015. 113 f. Dissertação (Mestrado) - Curso de Mestrado em Física, Universidade do Estado do Rio Grande do Norte, Mossoró, 2015.

[15] REBOUÇAS, Gustavo de Oliveira Gurgel. Nucleação de Vórtices e Paredes de Domínio em Nanoestruturas Magnéticas. 2010. 128 f. Tese (Doutorado) - Curso de Doutorado em Física, Universidade Federal do Rio Grande do Norte, Natal, 2010.

[16] GUIMARÃES, Alberto P.. Principles of Nanomagnetism. Rio de Janeiro: 2009.

[17] YOUNG, David A.. Phase Diagram of the Elemets. Berkeley: University Of California Press, 1991.

[18] REIS, M. Fundamentals of Magnetism. First edition. [S.1.]: Academic Press, 2013. ISBN 9780124055452. 